\title{
Edítorial.
}

\section{THEORIES OF SENSORY FUNCTION.}

A MONG the dwelling-places of neurological theory it is well from are apt to gain from the appearance of the superstructure a false sense of the solidity of the whole.

It is now nearly twenty years since the modern theory of cutaneous sensibility was founded upon the pioneer work of Head and Rivers.

Their method, involving experimental nerve section in the human subject, was new to medical science, and, it seems, must form the basis of future researches in this direction. Their observations were precisely recorded, and have in the main been confirmed by subsequent workers. Their interpretation of these records, and the theory of sensory function which they consequently deduced, have been widely accepted by physiologists, and have, moreover, served as a basis for further inquiries, which have reached their culmination in the attractive hypotheses set forth in Head's monograph on sensory disturbances in relation to lesions of the optic thalamus and of the cerebral cortex.

The central idea which runs through Head's later papers is the belief, deduced from the first experiments, that sensibility in the peripheral nerve involves the existence of two separate groups of nerve fibres, the one 'protopathic,' of a primitive quality, the other 'epicritic,' concerned mainly with the finer differentiation of stimuli, of a higher order, and of more recent development in the history of the race.

The explanation, in these terms, of the phenomena which accompany regeneration of the peripheral sensory nerve fibre is that in the first stage of regeneration the function of 'protopathic' fibres may be observed, released for the time being from 'epicritic' control. As the process of repair becomes completed, the process of evolution is briefly recapitulated. With the appearance of 'epicritic' sensibility, 'protopathic' function is submerged. According to this hypothesis, therefore, the occurrence at a certain state of repair of a certain abnormal state of sensibility is explained as a 'release phenomenon.' The same doctrine explains subsequent conclusions concerning the clinical results of higher lesions. The abnormalities of sensation often associated voL. v.-No. 18. 
with a lesion near the optic thalamus are ascribed to release of the thalamus from ' the restraining influence of the cortex.'

Neurology owes much to Hughlings Jackson's conception that ' positive' symptoms following a ' negative' lesion may be thus interpreted as release phenomena, but there is a danger of forgetting that, apart from complete destruction, a nerve cell may be so affected by disease as to react to stimuli in a positively abnormal manner.

Trotter and Davies, who repeated the work of Head and Rivers, were in the first place unable to confirm certain of the observations upon which the 'protopathic-epicritic' theory depended, and for the state of abnormal sensibility preceding complete regeneration they found a simpler explanation. They concluded that these abnormalities-which comprise quantitatively a raised threshold, qualitatively a reaction of pain or discomfort to all stimuli which pass that threshold-are due to the physiological state of the nerve fibres in the phase of incomplete repair. If this explanation be the correct one, Head's later hypotheses are in some danger of falling to the ground.

So far as the facts of clinical experience can avail in throwing light upon this problem they are worth examination.

As Trotter has again insisted in a recent review, the chief peculiarity of cutaneous sensibility in a recovering area is the intensification of sensations, which "gives to the sensation a kind of explosive vividness and brilliance which constitutes a wholly new experience." From the accounts given by patients we can recognize these qualities in certain other conditions. Of these the most striking is causalgia, a condition in which the peripheral nerve lesion is always incomplete. We meet them also in the case of lesions of the sensory pathway at any point within the central nervous system below the thalamus. The most important instances of this nature are those met with in early tabes, in incomplete traumatic lesions of the spinal cord, and certain cases of syringomyelia.

Here, then, is the same peculiar abnormality of sensation occurring as the result of lesions of various kinds at different levels of the afferent pathway-peripheral nerve, posterior nerve root, spinal cord, and brain-stem. The identity of the sensory experience in each case requires a single explanation. This in terms of Head's and Rivers' hypothesis is not readily available. In the case of the peripheral nerve and nerve root their explanation is release of protopathic fibres from epicritic control ; in the case of thalamic lesion, release of the thalamus from cortical control ; while for the occurrence of similar phenomena from lesions of the spinal cord no adequate explanation is apparently forthcoming.

Trotter's hypothesis, on the other hand, provides a single solution. According to this view the peculiar quality of sensation is due to an 
abnormal physiological state of nerve fibres, which are midway between destruction and repair, and this can at once be correlated with the fact that in the clinical conditions which we have mentioned the essential feature in each case is the incompleteness of the lesion.

We believe it possible that on account of its simpler applicability to clinical phenomena as well as to the facts of experimental observation, the Trotter-Davies hypothesis may eventually replace that of Head and Rivers among the foundations of neurological theory. 